\section{Against the oxidative damage theory of aging: superoxide dismutases protect against oxidative stress but have little or no effect on life span in Caenorhabditis elegans}

\author{
Ryan Doonan, ${ }^{1,3}$ Joshua J. McElwee, ${ }^{1,3}$ \\ Filip Matthijssens, ${ }^{2,3}$ Glenda A. Walker, ${ }^{1}$ \\ Koen Houthoofd, ${ }^{2}$ Patricia Back, ${ }^{2}$ \\ Andrea Matscheski, ${ }^{1}$ Jacques R. Vanfleteren, ${ }^{2}$ \\ and David Gems ${ }^{1,4}$

\begin{abstract}
${ }^{1}$ Institute of Healthy Ageing and Research Department of Genetics, Evolution and Environment, University College London, London WC1E 6BT, United Kingdom; ${ }^{2}$ Department of Biology, Ghent University, B-9000 Ghent, Belgium
\end{abstract}

The superoxide radical $\left(\mathrm{O}_{2}^{-}\right)$has long been considered a major cause of aging. $\mathrm{O}_{2}^{-}$in cytosolic, extracellular, and mitochondrial pools is detoxified by dedicated superoxide dismutase (SOD) isoforms. We tested the impact of each SOD isoform in Caenorhabditis elegans by manipulating its five sod genes and saw no major effects on life span. sod genes are not required for daf-2 insulin/ IGF-1 receptor mutant longevity. However, loss of the extracellular $\mathrm{Cu} / \mathrm{ZnSOD}$ sod-4 enhances daf-2 longevity and constitutive diapause, suggesting a signaling role for sod-4. Overall, these findings imply that $\mathrm{O}_{2}^{-}$is not a major determinant of aging in C. elegans.

Supplemental material is available at http://www.genesdev.org.

Received April 11, 2008; revised version accepted September 29,2008

Many forms of pathology lead to elevated levels of damage to biological macromolecules (Halliwell and Gutteridge 2007). This is also true of aging, the poorly understood biological process that leads to progressive deterioration and death. One strategy to discover the underlying mechanisms of aging has been to seek the causes of its associated molecular damage. An important early theory, proposed by Harman (1956), postulates that the cause might be oxygen free radicals. Harman later developed the theory, proposing a central role for the superoxide $\left(\mathrm{O}_{2}{ }^{-}\right)$radical, issuing from the mitochondrial electron transport chain (Harman 1972). During the last few decades, much effort has been invested in tests of this nexus of theories (for review, see Muller et al. 2007). Despite this, the importance of $\mathrm{O}_{2}{ }^{-}$as a cause of aging remains uncertain.

[Keywords: Aging, Caenorhabditis elegans; free radical; superoxide dismutase; insulin/IGF-1 signaling; genetics]

${ }^{3}$ These authors contributed equally to this work.

${ }^{4}$ Corresponding author.

E-MAIL david.gems@ucl.ac.uk; FAX 44-20-76797096.

Article is online at http://www.genesdev.org/cgi/doi/10.1101/gad.504808.
In this study, we take a genetic approach to critically test the role of $\mathrm{O}_{2}{ }^{-}$in aging in a short-lived animal, the nematode Caenorhabditis elegans, by manipulating expression of genes encoding the antioxidant enzyme superoxide dismutase (SOD). This enzyme catalyzes the dismutation reaction

$$
2 \mathrm{O}_{2}{ }^{-}+2 \mathrm{H}^{+} \rightarrow \mathrm{H}_{2} \mathrm{O}_{2}+\mathrm{O}_{2} .
$$

$\mathrm{H}_{2} \mathrm{O}_{2}$ (hydrogen peroxide) may then be broken down into $\mathrm{H}_{2} \mathrm{O}$ and $\mathrm{O}_{2}$ by catalase or glutathione peroxidase. $\mathrm{O}_{2}^{-}$ does not readily cross cellular membranes and, consequently, there are distinct extracellular, cytosolic, and mitochondrial $\mathrm{O}_{2}{ }^{-}$pools (Missirlis et al. 2003; Muller et al. 2004). In principle, one or more of these three $\mathrm{O}_{2}^{-}$ pools may play a role in aging.

Instrumental in lowering levels of $\mathrm{O}_{2}{ }^{-}$in each pool is a dedicated, compartment-specific SOD isoform. Cytosolic and extracellular $\mathrm{O}_{2}{ }^{-}$is consumed by distinct $\mathrm{Cu} /$ $\mathrm{ZnSOD}$ isoforms, while $\mathrm{O}_{2}{ }^{-}$in the mitochondrial matrix is consumed by MnSOD (Weisiger and Fridovich 1973). Most eukaryotes have a single SOD isoform for each compartment, but C. elegans, unusually, possesses two isoforms for each compartment. The two cytosolic $\mathrm{Cu} /$ ZnSOD isoforms are encoded by sod -1 and sod-5 (Larsen 1993; Giglio et al. 1994; Jensen and Culotta 2005). The sod-4 gene encodes two predicted extracellular $\mathrm{Cu} /$ ZnSOD isoforms, products of alternative splicing of mRNA (Fujii et al. 1998). Two mitochondrial MnSOD isoforms are encoded by sod-2 and sod-3 (Giglio et al. 1994; Suzuki et al. 1996; Hunter et al. 1997).

This superabundance of SOD isoforms has been a technical hurdle to investigations of the role of SOD and $\mathrm{O}_{2}^{-}$ in aging in C. elegans, and some of the sod genes have been barely studied. In situ gel SOD activity assays of a sod-1 deletion mutant imply that this gene encodes the major cytosolic Cu/ZnSOD (Jensen and Culotta 2005), leaving the function of sod-5 unclear. sod-3 mRNA levels are elevated in the dauer larva (Honda and Honda 1999), suggesting that this gene may play a special role in antioxidant defense in this long-lived, stress-resistant diapausal stage, but the role of sod-2 has remained obscure. In this study, we describe in detail the function of each of the five sod genes, characterizing their expression, and the phenotypic effects of manipulating their expression. This has allowed us to assess the effect on life history, especially aging, of each of the three major $\mathrm{O}_{2}^{-}$pools, thereby critically testing the role of SOD and, by inference, $\mathrm{O}_{2}{ }^{-}$, in longevity assurance and aging.

$\mathrm{O}_{2}{ }^{-}$can affect living organisms in a variety of ways. It can cause molecular damage that might contribute to aging; thus, one expectation of our study was that lowering SOD activity and increasing $\mathrm{O}_{2}{ }^{-}$levels might accelerate aging, and vice versa. $\mathrm{H}_{2} \mathrm{O}_{2}$ derived from $\mathrm{O}_{2}{ }^{-}$can also act a secondary messenger-for example, in receptor tyrosine kinase signaling pathways (Finkel 1998)—and as an activator of heat-shock factor. $\mathrm{O}_{2}^{-}$can also be deployed as a chemical weapon in immune defense against bacterial pathogens in higher animals, and probably in $C$. 
elegans as well (Chavez et al. 2007). There is even evidence that in C. elegans $\mathrm{O}_{2}{ }^{-}$can increase life span, perhaps by activating stress defense processes (Cypser and Johnson 2002; Schulz et al. 2007).

A powerful approach to investigate mechanisms of aging is the mutational analysis of genes with effects on life span. In C. elegans, mutations affecting the insulin/ IGF-1 signaling (IIS) pathway can strikingly increase adult life span. For example, mutation of daf-2, which encodes an insulin/IGF-1 receptor, can increase adult life span by more than twofold (Kenyon 2005). Severe daf-2 loss of function can also cause constitutive formation of dauer larvae, which are developmentally arrested, longlived, diapausal third-stage larvae (Riddle and Albert 1997).

One possibility is that increased SOD levels and reduced damage from $\mathrm{O}_{2}{ }^{-}$contribute to the longevity of IIS mutants. daf-2 mutants do show increased SOD and catalase activity levels, and resistance to oxidative stress (Vanfleteren 1993; Honda and Honda 1999). sod-3 mRNA and protein levels are elevated in daf-2 mutants (Honda and Honda 1999; Yanase et al. 2002; Dong et al. 2007), suggesting a possible role for mitochondrial MnSOD in longevity assurance. However, a more critical test is to examine the effects of alteration of SOD activity on life span.

In this study, we first examine the biology of the five C. elegans sod genes and show that sod-1 and sod-2 encode the major $\mathrm{Cu} / \mathrm{ZnSOD}$ and $\mathrm{MnSOD}$ isoforms in reproductive development, while sod-5 and sod-3 encode minor, auxiliary isoforms mainly expressed in dauer larvae. We then critically test the importance of SOD and, by extension, $\mathrm{O}_{2}{ }^{-}$in $C$. elegans aging and in the daf-2 Age phenotype by means of sod gene deletion and overexpression.

\section{Results and Discussion}

To understand the respective roles of the five sod genes, we characterized their expression using several techniques, including RT-PCR, Western blot analysis, SOD activity assays, and analysis of expression of sod::gfp transgenes. Expression was studied mainly in wild-type third-stage (L3) larvae and dauer larvae, daf-2(m577) mutants at the L3 stage, and mutants with deletions in each of the five sod genes (Supplemental Fig. S1A; for a detailed account of sod gene expression, see the Supplemental Material).

We report that sod-1 and sod-2 encode the major cytosolic $\mathrm{Cu} / \mathrm{ZnSOD}$ and MnSOD isoforms, respectively. sod-1 contributes $\sim 80 \%$ of total SOD activity and is ubiquitously expressed (Supplemental Fig. S4C), and SOD-1 protein is localized to the cytosol and mitochondrial intermembrane space (Fig. 3A, below). By contrast, sod-5 and sod-3 are, respectively, minor cytosolic $\mathrm{Cu} /$ ZnSOD and MnSOD isoforms whose expression is upregulated in dauer larvae (Supplemental Figs. S1B-E, S4D,F). In wild-type L3s, sod-5::gfp expression is largely restricted to the ASI, ASK, and ASG amphid neurons (Supplemental Fig. S3B,C), which influence longevity and dauer larva formation (Bargmann and Horvitz 1991; Alcedo and Kenyon 2004).

It has been shown previously that in daf-2 mutants, there is an elevation in levels of SOD activity (Vanfleteren 1993) and large fold increase in sod-3 mRNA (Honda and Honda 1999; Yanase et al. 2002). We confirmed this but also saw increases in expression of sod-1 and sod-5 (Supplemental Figs. S1B, S4B-D; Supplemental Material). Although sod-3 is the most highly up-regulated of the sod genes in terms of fold change in expression, its relative contribution to SOD levels remains very small, both in terms of overall SOD activity and MnSOD protein levels (Supplemental Fig. S1B-E).

We examined the organismal effects of deletion alleles of sod-2 and sod-3 MnSOD genes on C. elegans. We find that sod-2(0) but not sod-3(0) results in delayed development (data not shown), delayed and reduced reproduction, and a slowed defecation cycle (Supplemental Fig. S5). However, sod-3(0) further reduces fertility of sod2(0) animals (Supplemental Fig. S5B), implying functional redundancy between sod-2 and sod-3.

As expected, loss of MnSOD increases sensitivity to oxidative stress. sod-2(0) causes a moderate reduction in resistance to the $\mathrm{O}_{2}{ }^{-}$generator paraquat, while sod-3(0) does not, either alone or when added to sod-2(0) (Fig. 1A). In combination, sod-2(0) and sod-3(0) cause severe hypersensitivity to hyperoxia (60\% oxygen), while each mutation alone has no effect (Fig. 1B). Thus, sod-2 and sod-3 are functionally redundant when defending against mild but not severe oxidative stress.

Next, we tested the effect of sod-2(0) and sod-3(0) on life span. Mean life span is unaffected by either mutation alone (Fig. 1C; Supplemental Table S1) or both mutations combined (Fig. 1D; Supplemental Table S1). Thus, surprisingly, complete absence of MnSOD has no effect on life span. We also find that activity of cytosolic and
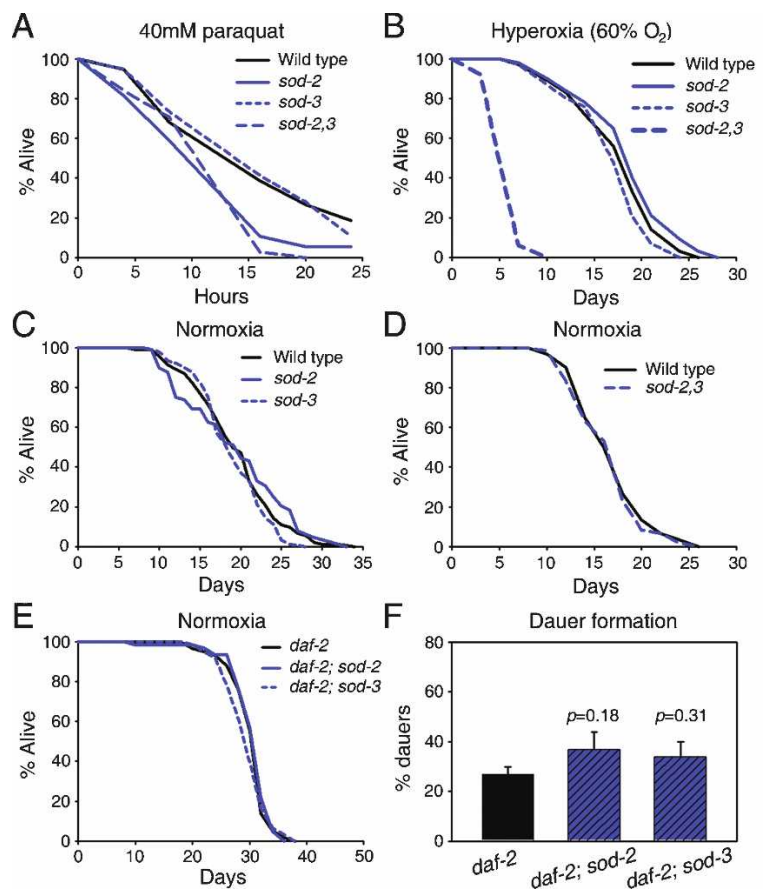

Figure 1. Oxidative stress resistance and longevity of MnSOD mutants. (A) Survival in $40 \mathrm{mM}$ paraquat. $(B)$ Survival under hyperoxia $\left(60 \% \mathrm{O}_{2}\right) \cdot(C, D)$ Life span of MnSOD single $(C)$ and double $(D) \mathrm{mu}-$ tants under normoxia $\left(20^{\circ} \mathrm{C}\right)$. (E) Effect of sod-2(0) or sod-3(0) on daf-2(m577) longevity $\left(25^{\circ} \mathrm{C}\right) .(F)$ Effect of sod-2(0) or sod-3(0) on daf-2(m577) Daf-c. 
mitochondrial aconitase (an oxidation-sensitive ironsulfur protein) is not detectably reduced in sod-2; sod-3 animals (data not shown), suggesting no major increase in oxidative damage to protein. To test for a role in $d a f-2$ mutant longevity, we examined the impact of sod-2(0) or sod-3(0) on daf-2 longevity but there is none (Fig. 1E; Supplemental Table S2). Altogether, these results strongly imply, against expectation, that mitochondrial matrix $\mathrm{O}_{2}^{-}$has no effect on aging and that MnSOD does not contribute to longevity assurance in C. elegans.

Next, we describe the effects of deletion of sod-1 and sod-5 cytosolic $\mathrm{Cu} / \mathrm{ZnSOD}$ genes. sod-1(0) increases sensitivity to paraquat, but sod-5(0) does not, either alone or when added to sod-1(0) (Fig. 2A). By contrast, neither sod-1(0) nor sod-5(0) has a marked effect on sensitivity to mild hyperoxia (Fig. 2B). sod-1(0) also decreases mean life span, by $15 \%-31 \%$, while sod $-5(0)$ has no effect, either alone or when added to sod-1(0) (Fig. 2C,D; Supplemental Table S1). Moreover, addition of sod-2(0) does not further reduce the life span of sod-1 mutants (data not shown), supporting the view that $\mathrm{O}_{2}{ }^{-}$does not move between mitochondrial and cytosolic pools. Potentially, sod-1(0) shortens life span by accelerating the age increase in molecular damage. We therefore examined the effect of sod-1(0) on the age increase in damage to protein and lipid but could not detect any acceleration (Supplemental Material), perhaps because the impact of sod-1(0) is relatively subtle and difficult to detect.

If the shorter life span of sod-1 mutants is due to accelerated aging, then overexpression of sod-1 should increase life span. To test this, we first examined the effect of expression of the $s o d-1:: g f p$ transgene on life span but
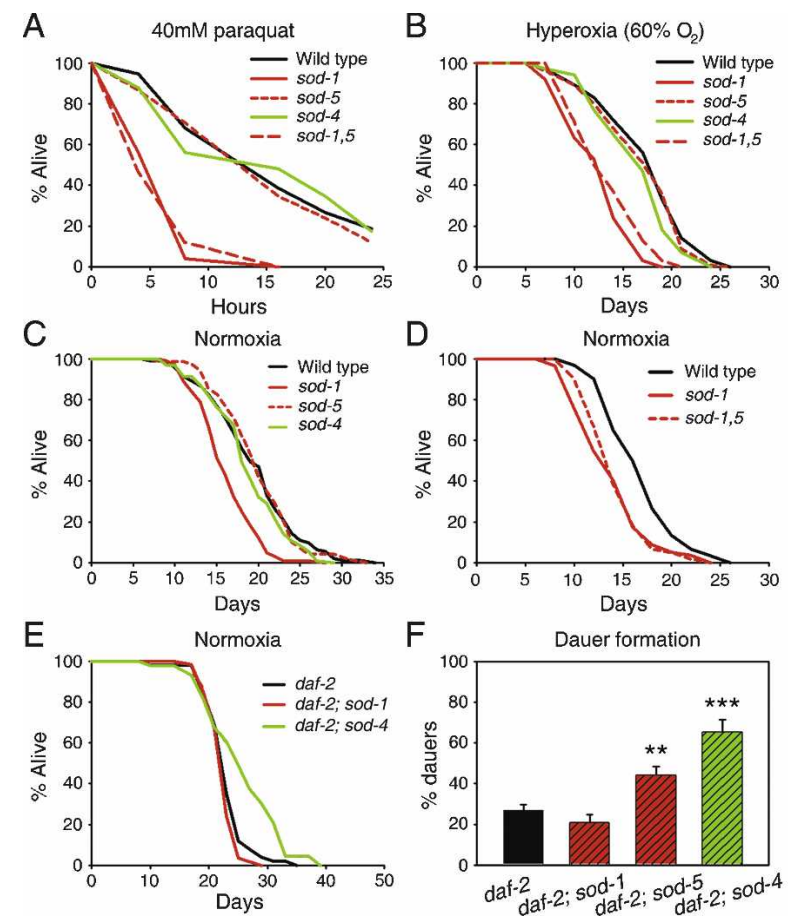

Figure 2. Oxidative stress resistance and longevity of $\mathrm{Cu} / \mathrm{ZnSOD}$ mutants. (A) Survival in $40 \mathrm{mM}$ paraquat. (B) Survival under hyperoxia $\left(60 \% \mathrm{O}_{2}\right) \cdot(C, D)$ Life span of $\mathrm{Cu} / \mathrm{ZnSOD}$ single $(C)$ and double $(D)$ mutants under normoxia $\left(20^{\circ} \mathrm{C}\right)$. (E) Effect of loss of $\mathrm{Cu} / \mathrm{ZnSOD}$ on daf-2(m577) longevity $\left(20^{\circ} \mathrm{C}\right)$. (F) Effect of loss of $\mathrm{Cu} / \mathrm{ZnSOD}$ on daf-2(m577) Daf-c. $\left(^{\star \star}\right) P<0.01 ;\left(^{\star \star \star}\right) P<0.0001$, Student's $t$-tests. saw no effect (data not shown). However, we subsequently discovered that fusion of GFP to SOD-1 reduces $\mathrm{Cu} / Z n S O D-s p e c i f i c$ activity (Supplemental Fig. S7; Supplemental Material). Next, we generated transgenic lines with multiple copies of the sod-1 gene, focusing initially on two lines bearing integrated transgene arrays, wuIs152 and wuIs154. Overall SOD activity is increased approximately twofold by wuIs152 (Fig. 3B) and wuIs154 (data not shown), and both lines show increased $\mathrm{Cu} / \mathrm{ZnSOD}$ immunoreactivity (Fig. 3A; data not shown).

Against expectation, sod-1 overexpression increases sensitivity to paraquat (Fig. 3E). Potentially, this could result from elevated levels of $\mathrm{H}_{2} \mathrm{O}_{2}$ due to faster conversion of paraquat-generated $\mathrm{O}_{2}{ }^{-}$into $\mathrm{H}_{2} \mathrm{O}_{2}$. To test this, we generated an integrated transgene array, wuIs151, with multiple copies of the entire ctl-1 ctl-2 ctl-3 gene cluster, which produces a 10 -fold increase in catalase activity (Fig. 3C). Catalase overexpression suppresses the increased paraquat sensitivity resulting from sod-1 overexpression (Fig. 3E), implying that this hypersensitivity is indeed due to elevated $\mathrm{H}_{2} \mathrm{O}_{2}$ levels.

wuIs 152 caused a statistically significant $(P<0.05)$ increase in life span in five out of 11 trials, and in no instance did it decrease life span (Supplemental Table S3). Combined data for these 11 trials defines a $21.5 \%$ increase in mean life span (Fig. 3F, $P<0.0001$ ). Although wuIs154 did not increase life span, increased life span was seen in three further lines with extrachromosomal arrays (wuEx125, wuEx122, and wuEx123) (Supplemental Table S3). From this we conclude that elevated SOD-1 can slightly increase life span and that the absence of an effect in wuIs154 likely reflects a life-shortening mutation associated with chromosomal insertion of the transgene array.

Given that SOD converts $\mathrm{O}_{2}{ }^{-}$into $\mathrm{H}_{2} \mathrm{O}_{2}$, it is possible that increased levels of SOD-1 lowers cytosolic $\mathrm{O}_{2}^{-}$but increases cytosolic $\mathrm{H}_{2} \mathrm{O}_{2}$, replacing damage from $\mathrm{O}_{2}$ with damage from $\mathrm{H}_{2} \mathrm{O}_{2}$. To test this, we compared the effect on life span of elevated SOD-1, catalase, or both. Overexpression of catalase alone results in a high level of mortality due to internal hatching of larvae (bagging) (Fig. 3D), perhaps reflecting $\mathrm{H}_{2} \mathrm{O}_{2}$ deficiency. This bagging is suppressed by overexpression of sod-1 (Fig. 3D), perhaps due to restoration of $\mathrm{H}_{2} \mathrm{O}_{2}$. In the absence of bagging (prevented by the inhibitor of DNA replication fluorodeoxyuridine, FUdR) overexpression of catalase slightly shortens life span, either alone or in addition to overexpression of sod-1 (Fig. 3G; SupplementalTable S3). Taken together, these results imply that cytosolic $\mathrm{Cu} /$ $\mathrm{ZnSOD}$ and, by implication, cytosolic $\mathrm{O}_{2}{ }^{-}$, are weak determinants of longevity and aging, respectively. By contrast, $\mathrm{H}_{2} \mathrm{O}_{2}$ does not seem to contribute to aging.

We next investigated whether sod-1 and sod-5 might contribute to the daf-2(m577) longevity (Age) phenotype. daf-2(m577) is a weak, temperature-sensitive (ts) allele resulting in a moderate increase in life span at $20^{\circ} \mathrm{C}$ and a large increase at $25^{\circ} \mathrm{C}$ (Gems et al. 1998; Patel et al. 2008). m577 was selected because it is a class 1 allele showing fewer pleiotropic effects than class 2 alleles such as e1370, making epistasis results easier to interpret. sod-1(0) slightly shortens daf-2 life span at $25^{\circ} \mathrm{C}$, perhaps reflecting a minor contribution of sod-1 to daf-2 Age, but not $20^{\circ} \mathrm{C}$ (Fig. 2E; Supplemental Table S2). sod-5(0) had little consistent effect (Supplemental Table S2). 
A

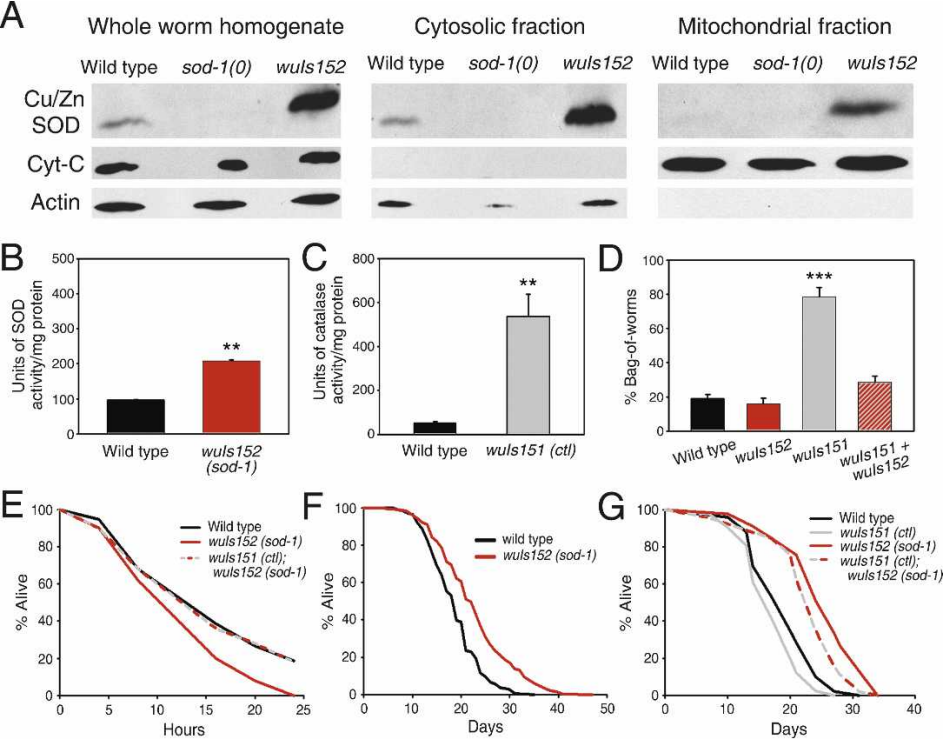

Figure 3. Overexpression of sod-1 cytosolic Cu/ZnSOD increases life span. $(A)$ Western blots of protein extracts of wild-type, sod-1 mutant, and sod-1 overexpresser lines using anti-Cu/ZnSOD antibodies. Cytochrome $\mathrm{C}(\mathrm{Cyt}-\mathrm{C})$ and actin were used as mitochondrion- and cytosol-specific markers, respectively. Increased SOD-1 protein was also detected in wuIs154 transgenics (data not shown). (B) Total SOD activity in protein extracts from lines overexpressing sod-1. $\left(^{\star \star}\right) P<0.01$, Student's $t$-test. $(C)$ Total catalase activity in line overexpressing catalase. $\left(^{\star \star}\right) P<0.01$, Student's $t$-test. $(D)$ Catalase overexpression causes high levels of mortality from internal hatching of larvae (bagging), and this is suppressed by overexpression of sod-1. $\left(^{\star \star \star} \mid P<0.0001\right.$, Student's $t$-test. (E) sod-1 overexpression increases sensitivity to oxidative stress $(40 \mathrm{mM}$ paraquat), and this is suppressed by overexpression of catalase. $(F)$ Overexpression of sod-1 increases life span. $(G)$ Elevated catalase does not further extend longevity of the sod-1 overexpresser.

Finally, we examined the phenotypic effects of loss of the sod-4 extracellular Cu/ZnSOD. sod-4(0) does not affect sensitivity to oxidative stress (Fig. 2A,B) or life span in otherwise wild-type animals (Fig. 2C). Surprisingly, sod-4(0) enhances daf-2 Age at both $20^{\circ} \mathrm{C}$ and $25^{\circ} \mathrm{C}$ (Fig. 2E; Supplemental Table S2). One possibility is that this reflects an effect on signaling. SOD-4, like mammalian $\mathrm{Cu} / \mathrm{ZnSOD}$, might generate $\mathrm{H}_{2} \mathrm{O}_{2}$, which then crosses into the cell and promotes insulin signaling by inhibiting redox-sensitive, signal-quenching phosphatases (Goldstein et al. 2005). In C. elegans, treatment with $\mathrm{H}_{2} \mathrm{O}_{2}$ increases $\mathrm{PIP}_{3}$ levels and promotes cytosolic retention of DAF-16 (Weinkove et al. 2006). If sod-4(0) does reduce IIS, then it should enhance other daf-2 mutant traits, including constitutive dauer larva formation (Daf-c). We therefore tested the effect of loss of each sod gene on daf-2(m577) Daf-c and report that sod-4(0) significantly enhances Daf-c (Figs. 1F, 2F). Thus, sod-4 may contribute to IIS.

$\mathrm{O}_{2}{ }^{-}$has long been viewed as a possible major determinant of aging (Harman 1956, 1972). In this study, we explored the importance of the three major $\mathrm{O}_{2}{ }^{-}$pools on aging in C. elegans. Overall, our results imply that $\mathrm{O}_{2}{ }^{-}$is not a major determinant of aging in this model organism, either in wild-type animals or long-lived daf-2(m577) mutants; however, it remains possible that SOD contributes substantially to longevity in other contexts (e.g., under dietary restriction). Our findings point to the novel conclusion that each $\mathrm{O}_{2}{ }^{-}$pool is different in terms of its effect on aging. In wild-type C. elegans, the cyto- solic $\mathrm{O}_{2}{ }^{-}$pool contributes weakly to aging while mitochondrial $\mathrm{O}_{2}{ }^{-}$, long considered a likely determinant of aging, and extracellular $\mathrm{O}_{2}{ }^{-}$have no detectable effect on normal aging. In daf-2 mutants, extracellular $\mathrm{O}_{2}^{-}$appears to promote longevity; however, it is more likely that SOD-4 converts $\mathrm{O}_{2}{ }^{-}$into $\mathrm{H}_{2} \mathrm{O}_{2}$, which then weakly activates IIS, thereby shortening life span. The effects of SOD and $\mathrm{O}_{2}{ }^{-}$on aging therefore vary according to cell compartment and to genotype (summarized in Fig. 4).

Our findings paint a clearer picture of the role of the various SOD isoforms in C. elegans. sod-1 and sod-2 encode the major $\mathrm{Cu} / \mathrm{ZnSOD}$ and MnSOD isoforms, corresponding to the equivalent isoforms in other eukaryotes. By contrast, sod-5 and sod-3 encode inducible, auxiliary $\mathrm{Cu} /$ $\mathrm{ZnSOD}$ and MnSOD isoforms. The presence of these supernumerary isoforms, like that of the stress-resistant dauer larva stage in which they are up-regulated, may reflect the hostile soil environment in which C. elegans has evolved.

We examined the effect on life span of loss of SOD in each of the three major cellular compartments. The oxidative damage theory of aging predicts that loss of SOD should cause accelerated aging, particularly cytosolic $\mathrm{Cu} / \mathrm{ZnSOD}$ and MnSOD, both of which contribute to scavenging of mitochondrially generated $\mathrm{O}_{2}^{-}$(the former in the mitochondrial intermembrane space). In fact, only loss of sod-1 shortened life span, and then only modestly. However, overexpression of sod-1 did increase life span slightly (Fig. 3F; Supplemental Table S3), implying a small contributory role of $\mathrm{O}_{2}^{-}$to aging.

Loss of MnSOD isoforms had no effect on life span, either in a daf-2(+) or daf-2(m577) background (Fig. 1C-E; Supplemental Tables S1, S2). This strongly implies that $\mathrm{O}_{2}{ }^{-}$within the mitochondrial matrix is not a significant cause of aging in C. elegans. An alternative possibility is that other mechanisms protect $C$. elegans mitochondria against $\mathrm{O}_{2}{ }^{-}$; however, the oxygen hypersensitivity of sod-2; sod-3 mutants argues against this.

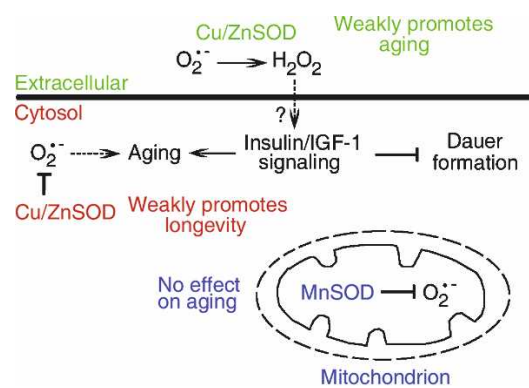

Figure 4. Influence of $\mathrm{SOD}$ and $\mathrm{O}_{2}{ }^{-}$on aging. This scheme shows a synthesis of conclusions drawn from the present study. Different SOD isoforms (and by deduction, the corresponding $\mathrm{O}_{2}^{-}$pools) have different effects on aging. Extracellular $\mathrm{Cu} / \mathrm{ZnSOD}$ weakly inhibits dauer formation and promotes aging, potentially by generating $\mathrm{H}_{2} \mathrm{O}_{2}$, which crosses into the cell and stimulates insulin/IGF-1 signaling by inhibiting redox-sensitive phosphatases. Cytosolic $\mathrm{Cu} /$ ZnSOD weakly promotes longevity, perhaps by protecting against molecular damage (sod-1 does not influence daf-2 Daf-c). Mitochondrial MnSOD has no detectable effect on aging. Arrow with dotted line implies a weak effect. 
Very recently, another study also reported, using different mutant alleles, that sod-2(0); sod-3(0) does not affect life span in an otherwise wild-type background (Honda et al. 2008), confirming our findings. These investigators also observed that in a daf-2(e1370) mutant, sod-2(0) slightly shortened life span and lessened Daf-c while sod-3(0) had the opposite effect. daf-2 alleles fall into two phenotypic classes: Class 2 alleles are more pleiotropic than class 1 alleles and show more complex epistatic interactions with other mutations (Gems et al. 1998; Patel et al. 2008). The difference in effects of loss of MnSOD on daf-2(m577) (this study) and daf-2(e1370) (Honda et al. 2008) is interesting since $m 577$ is a class 1 allele and $e 1370$ a class 2 allele and suggests that MnSOD exerts a selective influence on class 2 -specific defects.

Loss of function of many genes involved in mitochondrial function result in a Clk or Mit phenotype, which includes a delayed reproductive schedule, lowered fertility, slowed defecation cycle, and increased life span (Wong et al. 1995; Rea 2005). In this study, we found that sod-2(0) results in all these traits (Supplemental Fig. S5) except the increase in life span (Fig. 1C,D). RNAi of sod-2 enhances clk-1(qm30) mutant longevity (Yang et al. 2007), suggesting a possible cryptic effect of sod-2 on aging via a Clk/Mit-type mechanism.

Loss of sod-4 enhances the daf-2 Age and Daf-c phenotypes (Fig. 2E,F), suggesting that sod-4 may play a role in IIS regulation of dauer formation and life span. We postulate that SOD-4-generated $\mathrm{H}_{2} \mathrm{O}_{2}$ promotes IIS by inhibiting IIS antagonistic phosphatase enzymes, as occurs in mammals (Goldstein et al. 2005).

While our findings imply that SOD and $\mathrm{O}_{2}{ }^{-}$have, at most, minor effects on aging in C. elegans, the question remains: What is the relevance of these findings to higher animals? The effects on life span of altering SOD gene expression vary between $C$. elegans, Drosophila, and the mouse (for review, see Muller et al. 2007). Loss of MnSOD causes early lethality in the fly and the mouse but not the worm, while loss of cytosolic $\mathrm{Cu} / \mathrm{ZnSOD}$ causes only small decreases life span in the worm and the mouse but a large $(\sim 80 \%)$ decrease in life span in the fly. Overexpressing cytosolic $\mathrm{Cu} / \mathrm{ZnSOD}$ slightly increases life span in C. elegans (this study), and in Drosophila overexpression of cytosolic $\mathrm{Cu} / \mathrm{ZnSOD}$ and MnSOD seem to increase life span, although findings vary (Muller et al. 2007); however, overexpression of cytosolic $\mathrm{Cu} / \mathrm{ZnSOD}$ does not increase life span in the mouse (Huang et al. 2000). By contrast, in the filamentous fungus Podospora anserina, reduction of mitochondrial ROS production results in a dramatic retardation of aging: Instead of aging rapidly after a brief period of growth, hyphae grow continuously and, apparently, indefinitely (Dufour et al. 2000).

It has been suggested that damage from reactive oxygen species might represent a public (i.e., evolutionarily conserved) rather than a private (i.e., lineage-specific) mechanism of aging (Martin et al. 1996). Our findings imply that the role of $\mathrm{O}_{2}^{-}$in aging is to some extent public and to some extent private: Cytosolic $\mathrm{O}_{2}{ }^{-}$appears to contribute to aging in C. elegans and Drosophila but not mice. By contrast, loss of MnSOD is lethal to the fly and the mouse but has no effect on aging in the worm. Thus, $\mathrm{O}_{2}{ }^{-}$in some cellular compartments seems to contribute to some degree to aging in some species, but in other contexts (e.g., C. elegans) it appears unimportant to aging.

\section{Materials and methods}

Nematode culture

Nematodes were cultured on NGM agar seeded with E. coli OP50 as described previously (Sulston and Hodgkin 1988). Strains were maintained at $20^{\circ} \mathrm{C}$ unless otherwise noted. Mutant alleles and transgenic arrays used or generated in this study are listed in the Supplemental Material.

Oxidative stress resistance assays

For paraquat, young adults were placed overnight on agar plates containing $40 \mu \mathrm{M}$ fluorodeoxyuridine (FUdR), and then picked into microtiter wells with $100 \mu \mathrm{l}$ of $40 \mathrm{mM}$ paraquat (Sigma, 856177) in M9 buffer. Viability was assayed over a 20 -h period. For hyperoxia, young adults were picked onto plates containing $40 \mu \mathrm{M}$ FUdR and placed in a sealed chamber under $60 \% \mathrm{O}_{2}\left(22^{\circ} \mathrm{C}\right)$. Animals were briefly removed from the $\mathrm{O}_{2}$ chamber to score viability every $2-3 \mathrm{~d}$.

\section{Life span measurements}

Life span assays were performed as described previously (Gems et al. 1998), at either $20^{\circ} \mathrm{C}$ or $25^{\circ} \mathrm{C}$ (see Supplemental Tables S1-S3). Survivorship of populations was compared statistically using the log rank test, performed using JMP 7.0.1 (SAS).

\section{Construction of transgenic lines}

Reporter constructs for each of the sod genes are full-length, translational fusions of GFP to the C terminus (see Supplemental Figure S2). For sod and $c t l$ (catalase) overexpression, gDNA fragments were generated by PCR and microinjected directly. For primer sequences for reporter and overexpression constructs, see the Supplemental Material. Unless otherwise noted, pRF4 [rol-6(su1006)] was used as a marker of transformation. Integrated lines were generated by X-irradiation, and backcrossed to wild type (N2) at least five times before further study.

SOD and catalase activity assays

SOD activity was measured using an assay involving the inhibition of superoxide-induced lucigenin chemiluminescence by SOD, as described previously (Lenaerts et al. 2002). Catalase activity was assayed at $25^{\circ} \mathrm{C}$ according to a standard method (Aebi 1984), adapted for use in microtiter plate format.

\section{Dauer formation assays}

Eggs from strains bearing daf-2(m577) were collected by hypochlorite lysis and cultured at $23.5^{\circ} \mathrm{C}$. The proportion of dauer larvae was scored $72 \mathrm{~h}$ later.

\section{Acknowledgements}

We thank David Hoogewijs for assistance with mRNA level estimations, and Peter Piper, Jennifer Tullet, and David Weinkove for useful discussion and comments on the manuscript. This work was supported by grants from the Wellcome Trust and the European Union (to D.G.), and Ghent University (GOA 12050101), the Fund for Scientific ResearchFlanders (G.0025.06), and the European Community (LSHM-CT-2004512020) (to J.R.V.). P.B. acknowledges a grant from the Institute for the Promotion of Innovation by Science and Technology in Flanders (IWT). Some strains were obtained from the Caenorhabditis Genetics Center, which is supported by the National Institutes of Health National Center for Research Resources.

\section{References}

Aebi, H. 1984. Catalase in vitro. Methods Enzymol. 105: 121-126.

Alcedo, J. and Kenyon, C. 2004. Regulation of C. elegans longevity by specific gustatory and olfactory neurons. Neuron 41: 45-55.

Bargmann, C.I. and Horvitz, H.R. 1991. Control of larval development by chemosensory neurons in Caenorhabditis elegans. Science 251: 1243-1246.

Chavez, V., Mohri-Shiomi, A., Maadani, A., Vega, L.A., and Garsin, D.A. 2007. Oxidative stress enzymes are required for DAF-16-mediated immunity due to generation of reactive oxygen species by Caenorhabditis elegans. Genetics 176: 1567-1577.

Cypser, J.R. and Johnson, T.E. 2002. Multiple stressors in Caenorhabditis 
elegans induce stress hormesis and extended longevity. J. Gerontol. 57: B109-B114.

Dong, M.Q., Venable, J.D., Au, N., Xu, T., Park, S.K., Cociorva, D., Johnson, J.R., Dillin, A., and Yates III, J.R. 2007. Quantitative mass spectrometry identifies insulin signaling targets in C. elegans. Science 317: 660-663.

Dufour, E., Boulay, J., Rincheval, V., and Sainsard-Chanet, A. 2000. A causal link between respiration and senescence in Podospora anserina. Proc. Natl. Acad. Sci. 97: 4138-4143.

Finkel, T. 1998. Oxygen radicals and signaling. Curr. Opin. Cell Biol. 10: 248-253.

Fujii, M., Ishii, N., Joguchi, A., Yasuda, K., and Ayusawa, D. 1998. Novel superoxide dismutase gene encoding membrane-bound and extracellular isoforms by alternative splicing in Caenorhabditis elegans. DNA Res. 5: 25-30.

Gems, D., Sutton, A.J., Sundermeyer, M.L., Larson, P.L., Albert, P.S., King, K.V., Edgley, M., and Riddle, D.L. 1998. Two pleiotropic classes of daf-2 mutation affect larval arrest, adult behavior, reproduction and longevity in Caenorhabditis elegans. Genetics 150: 129-155.

Giglio, M.-P., Hunter, T., Bannister, J.V., Bannister, W.H., and Hunter, G.J. 1994. The manganese superoxide dismutase gene of Caenorhabditis elegans. Biochem. Mol. Biol. Int. 33: 37-40.

Goldstein, B.J., Mahadev, K., and Wu, X. 2005. Redox paradox: Insulin action is facilitated by insulin-stimulated reactive oxygen species with multiple potential signaling targets. Diabetes 54: 311-321.

Halliwell, B. and Gutteridge, J.M.C. 2007. Free radicals in biology and medicine. Oxford University Press, Oxford, UK.

Harman, D. 1956. Aging: A theory based on free radical and radiation chemistry. J. Gerontol. 11: 298-300.

Harman, D. 1972. The biologic clock: The mitochondria? J. Am. Geriatr. Soc. 20: $145-147$.

Honda, Y. and Honda, S. 1999. The daf-2 gene network for longevity regulates oxidative stress resistance and Mn-superoxide dismutase gene expression in Caenorhabditis elegans. FASEB J. 13: 1385-1393.

Honda, Y., Tanaka, M., and Honda, S. 2008. Modulation of longevity and diapause by redox regulation mechanisms under the insulin-like signaling control in Caenorhabditis elegans. Exp. Gerontol. 43: 520529.

Huang, T., Carlson, E., Gillespie, A., Shi, Y., and Epstein, C. 2000. Ubiquitous overexpression of CuZn superoxide dismutase does not extend life span in mice. J. Gerontol. 55: B5-B9.

Hunter, T., Bannister, W.H., and Hunter, G.J. 1997. Cloning, expression, and characterization of two manganese superoxide dismutases from Caenorhabditis elegans. J. Biol. Chem. 272: 28652-28659.

Jensen, L.T. and Culotta, V.C. 2005. Activation of CuZn superoxide dismutases from Caenorhabditis elegans does not require the copper chaperone CCS. J. Biol. Chem. 280: 41373-41379.

Kenyon, C. 2005. The plasticity of aging: Insights from long-lived mutants. Cell 120: 449-460.

Larsen, P.L. 1993. Aging and resistance to oxidative stress in Caenorhabditis elegans. Proc. Natl. Acad. Sci. 90: 8905-8909.

Lenaerts, I., Braeckman, B., Matthijssens, F., and Vanfleteren, J. 2002. A high-throughput microtiter plate assay for superoxide dismutase based on lucigenin chemiluminescence. Anal. Biochem. 311: 90-92.

Martin, G.M., Austad, S.N., and Johnson, T.E. 1996. Genetic analysis of ageing: Role of oxidative damage and environmental stresses. Nat. Genet. 13: 25-34.

Missirlis, F., Hu, J., Kirby, K., Hilliker, A., Rouault, T., and Phillips, J. 2003. Compartment-specific protection of iron-sulfur proteins by superoxide dismutase. J. Biol. Chem. 278: 47365-47369.

Muller, F.L., Liu, Y., and Van Remmen, H. 2004. Complex III releases superoxide to both sides of the inner mitochondrial membrane. J. Biol. Chem. 279: 49064-49073.

Muller, F.L., Lustgarten, M.S., Jang, Y., Richardson, A., and Van Remmen, H. 2007. Trends in oxidative aging theories. Free Radic. Biol. Med. 43: 477-503.

Patel, D.S., Garza-Garcia, A., Nanji, M., McElwee, J.J., Ackerman, D., Driscoll, P.C., and Gems, D. 2008. Clustering of genetically defined allele classes in the Caenorhabditis elegans DAF-2 insulin/IGF-1 receptor. Genetics 178: 931-946.

Rea, S.L. 2005. Metabolism in the Caenorhabditis elegans Mit mutants. Exp. Gerontol. 40: 841-849.

Riddle, D.L. and Albert, P.S., eds. 1997. Genetic and environmental regu- lation of dauer larva development. Cold Spring Harbor Laboratory Press, Cold Spring Harbor, NY.

Schulz, T.J., Zarse, K., Voigt, A., Urban, N., Birringer, M., and Ristow, M. 2007. Glucose restriction extends Caenorhabditis elegans life span by inducing mitochondrial respiration and increasing oxidative stress. Cell Metab. 6: 280-293.

Sulston, J. and Hodgkin, J. 1988. Methods. In The Nematode Caenorhab ditis elegans (ed. W.B. Wood), pp. 587-606. Cold Spring Harbor Laboratory Press, Cold Spring Harbor, NY.

Suzuki, N., Inokuma, K., Yasuda, K., and Ishii, N. 1996. Cloning, sequencing and mapping of a manganese superoxide dismutase gene of the nematode Caenorhabditis elegans. DNA Res. 3: 171-174.

Vanfleteren, J.R. 1993. Oxidative stress and ageing in Caenorhabditis elegans. Biochem. J. 292: 605-608.

Weinkove, D., Halstead, J.R., Gems, D., and Divecha, N. 2006. Long-term starvation and ageing induce AGE-1/PI 3-kinase-dependent translocation of DAF-16/FOXO to the cytoplasm. BMC Biol. 4: 1. doi: 10.1186/1741-7007-4-1.

Weisiger, R.A. and Fridovich, I. 1973. Superoxide dismutase. Organelle specificity. J. Biol. Chem. 248: 3582-3592.

Wong, A.E., Boutis, P., and Hekimi, S. 1995. Mutations in the clk-1 gene of Caenorhabditis elegans affect developmental and behavioral timing. Genetics 139: 1247-1259.

Yanase, S., Yasuda, K., and Ishii, N. 2002. Adaptive responses to oxidative damage in three mutants of Caenorhabditis elegans lage-1, mev-1 and daf-16) that affect life span. Mech. Ageing Dev. 123: 15791587.

Yang, W., Li, J., and Hekimi, S. 2007. A measurable increase in oxidative damage due to reduction in superoxide detoxification fails to shorten the life span of long-lived mitochondrial mutants of Caenorhabditis elegans. Genetics 177: 2063-2074. 


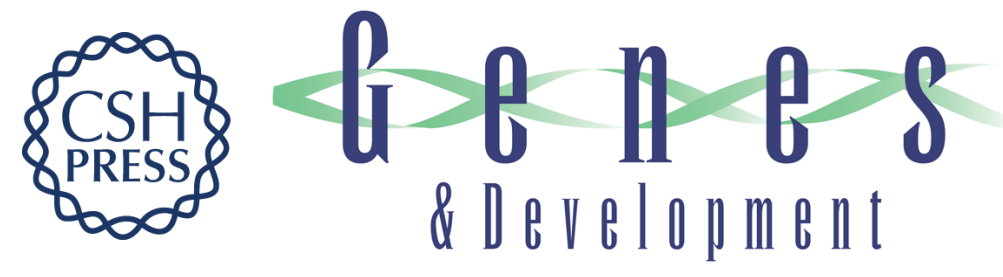

\section{Against the oxidative damage theory of aging: superoxide dismutases protect against oxidative stress but have little or no effect on life span in Caenorhabditis elegans}

Ryan Doonan, Joshua J. McElwee, Filip Matthijssens, et al.

Genes Dev. 2008, 22:

Access the most recent version at doi:10.1101/gad.504808

Supplemental Material

References License

Email Alerting Service
http://genesdev.cshlp.org/content/suppl/2008/11/18/22.23.3236.DC1

This article cites 36 articles, 16 of which can be accessed free at: http://genesdev.cshlp.org/content/22/23/3236.full.html\#ref-list-1

Receive free email alerts when new articles cite this article - sign up in the box at the top right corner of the article or click here.

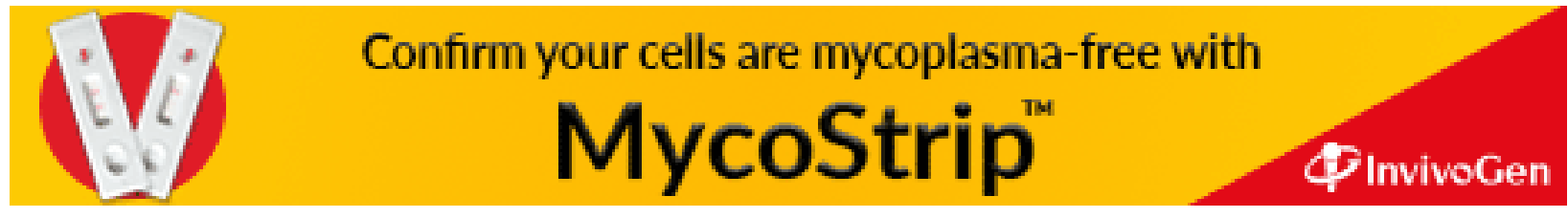

